VOL. 70 (2004) [489-497]

\title{
A NOTE ON A CONTINUED FRACTION OF RAMANUJAN
}

\author{
C. Adiga and N. Anitha
}

Ramanujan recorded many beautiful continued fractions in his notebooks. In this paper, we derive several identities involving the Ramanujan continued fraction $c(q)$, including relations between $c(q)$ and $c\left(q^{n}\right)$. We also obtain explicit evaluations of $c\left(e^{-\pi \sqrt{n}}\right)$ for various positive integers $n$.

\section{INTRODUCTION}

The celebrated Rogers-Ramanujan continued fraction is defined by

$$
R(q):=\frac{q^{1 / 5}}{1+} \quad \frac{q}{1+} \quad \frac{q^{2}}{1+} \quad \frac{q^{3}}{1+\cdots}, \quad|q|<1 .
$$

In his first two letters to Hardy [12, pp. xxvii, xxviii], Ramanujan communicated several theorems about $R(q)$ and $S(q):=-R(-q)$. In these two letters, Ramanujan claimed that

$$
R\left(e^{-2 \pi}\right)=\sqrt{\frac{5+\sqrt{5}}{2}}-\frac{\sqrt{5}+1}{2}
$$

and

$$
S\left(e^{-\pi}\right)=\sqrt{\frac{5-\sqrt{5}}{2}}-\frac{\sqrt{5}-1}{2} .
$$

Ramanujan recorded other values for $R(q)$ and $S(q)$ in his first notebook [11] and in his 'lost' notebook [13]. Most of these results were proved by Ramanathan [8, 9, 10], Berndt and Chan [5]. On page 365 of his 'lost' notebook, Ramanujan wrote five modular equations relating $R(q)$ with $R(-q), R\left(q^{2}\right), R\left(q^{3}\right), R\left(q^{4}\right)$ and $R\left(q^{5}\right)$. Ramanujan eventually found several generalisations and ramifications of (1.1) which are recorded in his 'lost' notebook.

Recently, Berndt and Chan [5], Chan[6], Chan and Huang [7], Adiga, Vasuki and Naika[2] and Adiga, Vasuki and Shivashankara [3] have established several new interesting evaluations of the Rogers-Ramanujan continued fraction, Ramanujan's cubic continued fraction, and the Ramanujan-Göllnitz-Gordon continued fraction.

Motivated by these works, in this paper we study the Ramanujan continued fraction

$$
c(q):=\frac{1}{1+} \quad \frac{2 q}{1-q^{2}+} \quad \frac{q^{2}\left(1+q^{2}\right)^{2}}{1-q^{6}+} \quad \frac{q^{4}\left(1+q^{4}\right)^{2}}{1-q^{10+} \ldots}, \quad|q|<1 .
$$

Received 19th July, 2004

Copyright Clearance Centre, Inc. Serial-fee code: 0004-9727/04 \$A2.00+0.00. 
In Section 2, we obtain a product representation for $c(q)$. In Section 3, we deduce some basic identities satisfied by $c(q)$ and also establish relations between $c(q)$ and $c(-q)$ and $c(q)$ and $c\left(q^{2}\right)$.

In Section 4, we derive relations between $c(q)$ and $c\left(q^{n}\right)$ using modular equations of degree $\mathrm{n}$. In the final section we shall establish two formulas for evaluating $c\left(e^{-\pi \sqrt{n}}\right)$ in terms of Ramanujan-Weber class invariants. We use these formulas to evaluate explicitly $c\left(e^{-\pi \sqrt{n}}\right)$ for various positive integers $n$.

\section{Product Representation For $c(q)$}

THEOREM 2.1. Let $c(q)$ be as defined in (1.2). Then,

$$
c(q)=\frac{\phi\left(q^{4}\right)}{\phi(q)}
$$

where

$$
\phi(q)=\sum_{k=-\infty}^{\infty} q^{k^{2}}=\frac{(-q ;-q)_{\infty}}{(q ;-q)_{\infty}}
$$

and

$$
(a)_{\infty}=(a ; q)_{\infty}:=\prod_{n=0}^{\infty}\left(1-a q^{n}\right) .
$$

Proof: From Entry 11 of $[1$, p. 14], we have

$$
\frac{(-a)_{\infty}(b)_{\infty}-(a)_{\infty}(-b)_{\infty}}{(-a)_{\infty}(b)_{\infty}+(a)_{\infty}(-b)_{\infty}}=\frac{(a-b)}{1-q+} \quad \frac{(a-b q)(a q-b)}{1-q^{3}+} \quad \frac{q\left(a-b q^{2}\right)\left(a q^{2}-b\right)}{1-q^{5}+\cdots}
$$

which can be rewritten as

$$
1+\frac{(-a)_{\infty}(b)_{\infty}}{(a)_{\infty}(-b)_{\infty}}=\frac{2}{1-} \quad \frac{(a-b)}{1-q+} \quad \frac{(a-b q)(a q-b)}{1-q^{3}+} \quad \frac{q\left(a-b q^{2}\right)\left(a q^{2}-b\right)}{1-q^{5}+\cdots} .
$$

Changing $q$ to $q^{2}, a$ to $z q$ and $b$ to $-q / z$ in (2.1) we obtain

$$
1+\frac{\left(-z q ; q^{2}\right)_{\infty}\left(-q / z ; q^{2}\right)_{\infty}}{\left(z q ; q^{2}\right)_{\infty}\left(q / z ; q^{2}\right)_{\infty}}=\frac{2}{1-} \quad \frac{z q+q / z}{1-q^{2}+} \quad \frac{\left(z q+q^{3} / z\right)\left(z q^{3}+q / z\right)}{1-q^{6}+\cdots}
$$

Employing Entry 30(ii) [1, p. 43] in (2.2) we deduce that

$$
\frac{f\left(z^{2} q^{4}, q^{4} / z^{2}\right)}{f(-z q,-q / z)}=\frac{1}{1-} \quad \frac{z q+q / z}{1-q^{2}+} \quad \frac{\left(z q+q^{3} / z\right)\left(z q^{3}+q / z\right)}{1-q^{6}+\cdots}
$$

where

$$
f(a, b)=\sum_{k=-\infty}^{\infty} a^{k(k+1) / 2} b^{k(k-1) / 2}=(-a ; a b)_{\infty}(-b ; a b)_{\infty}(a b ; a b)_{\infty}
$$

Putting $z=-1$ in (2.3) and noting that $f(q, q)=\phi(q)$, we complete the proof. 


\section{Some Identities INVOlving $c(q)$}

In Chapter 16 of his second notebook [11], Ramanujan develops the theory of Thetafunction using his function $f(a, b)$ and its restrictions

$$
\phi(q):=f(q, q)=\frac{(-q ;-q)_{\infty}}{(q ;-q)_{\infty}}
$$

and

$$
\psi(q):=f\left(q ; q^{3}\right)=\frac{\left(q^{2} ; q^{2}\right)_{\infty}}{\left(q ; q^{2}\right)_{\infty}} .
$$

Now we derive some identities involving $c(q)$.

THEOREM 3.1. We have,

(i) $c^{2}(q)=\frac{\psi^{4}\left(q^{4}\right)}{\phi^{2}(q) \psi^{2}\left(q^{8}\right)}$,

(ii) $c^{2}(q)-c^{2}(-q)=\frac{-8 q \psi^{6}\left(q^{4}\right)}{\psi^{2}\left(q^{8}\right) \phi^{4}\left(-q^{4}\right)}$,

(iii) $c^{2}(q) \cdot c^{2}(-q)=\frac{\phi^{4}\left(q^{4}\right)}{\phi^{4}\left(-q^{2}\right)}$, and

(iv) $\frac{c^{-1}(q)-c(q)}{c^{-1}(q)+c(q)}=\frac{\phi^{2}(q)-\phi^{2}\left(q^{4}\right)}{\phi^{2}(q)+\phi^{2}\left(q^{4}\right)}$.

Proof of (i): We have

$$
c(q)=\frac{\phi\left(q^{4}\right)}{\phi(q)} .
$$

Also from [1, p. 36, Entry 25(iv)], we have

$$
\phi(q)=\frac{\psi^{2}(q)}{\psi\left(q^{2}\right)}
$$

and

$$
\phi\left(q^{4}\right)=\frac{\psi^{2}\left(q^{4}\right)}{\psi\left(q^{8}\right)} .
$$

Substituting (3.2) in (3.1) and squaring, we deduce that

$$
c^{2}(q)=\frac{\psi^{4}\left(q^{4}\right)}{\phi^{2}(q) \psi^{2}\left(q^{8}\right)} .
$$

This completes the proof of (i).

ProOF OF (ii): From (i) we have,

$$
c^{2}(q)=\frac{\psi^{4}\left(q^{4}\right)}{\phi^{2}(q) \psi^{2}\left(q^{8}\right)} .
$$


Replacing $q$ by $-q$, we obtain

$$
c^{2}(-q)=\frac{\psi^{4}\left(q^{4}\right)}{\phi^{2}(-q) \psi^{2}\left(q^{8}\right)} .
$$

Taking the difference of the above two equations and using [1, p. 36, Entry 25(iii), (v)], we complete the proof.

Proof OF (iii): The result follows from (i) and [1, p. 36, Entry 25 (iii) and (iv)].

Proof of (iv): By Theorem 2.1, we deduce that

$$
c^{-1}(q)-c(q)=\frac{\phi^{2}(q)-\phi^{2}\left(q^{4}\right)}{\phi(q) \phi\left(q^{4}\right)}
$$

and

$$
c^{-1}(q)+c(q)=\frac{\phi^{2}(q)+\phi^{2}\left(q^{4}\right)}{\phi(q) \phi\left(q^{4}\right)} .
$$

On dividing (3.3) by (3.4) we complete the proof.

REMARK: Identities in Theorem 2.1 are similar to those identities satisfied by $H(q)$ in [7, Theorem 2.1].

THEOREM 3.2. Let $u=c(q), v=c(-q)$ and $w=c\left(q^{2}\right)$. Then,

(i) $u+v=2 u v$,

(ii) $u=\frac{1}{2}\left(\frac{1+\sqrt{1-(2 w-1)^{4}}}{(2 w-1)^{2}}+1\right)$, and

(iii) $(u-v)^{2}(2 w-1)^{2}+8 u v w(w-1)=0$.

Proof of (i): Follows easily from Theorem 2.1 and [1, Entry 25(i), p. 35].

Proof of (ii): We have,

$$
w=\frac{\phi\left(q^{2}\right)+\phi\left(-q^{2}\right)}{2 \phi\left(q^{2}\right)}
$$

and hence

$$
(2 w-1)^{2}=\frac{\phi^{2}\left(-q^{2}\right)}{\phi^{2}\left(q^{2}\right)} .
$$

From [1, p. 36, Entry 25 (iii), (vi)], we have

$$
(2 w-1)^{2}=\frac{2(\phi(-q) / \phi(q))}{1+\left(\phi^{2}(-q) / \phi^{2}(q)\right)} .
$$

Thus,

$$
(2 w-1)^{2}=\frac{2(2 u-1)}{1+(2 u-1)^{2}} .
$$

On simplification, we obtain the desired result. 
Proof OF (iii): We have,

$$
\begin{aligned}
& u=\frac{\phi(q)+\phi(-q)}{2 \phi(q)}, \\
& v=\frac{\phi(q)+\phi(-q)}{2 \phi(-q)},
\end{aligned}
$$

and hence

$$
\frac{u}{v}=\frac{\phi(-q)}{\phi(q)}
$$

From (3.5), we have

$$
(2 w-1)^{2}=\frac{2(u / v)}{1+\left(u^{2} / v^{2}\right)}=\frac{2 u v}{u^{2}+v^{2}} .
$$

On simplification, we obtain,

$$
(u-v)^{2}(2 w-1)^{2}+8 u v w(w-1)=0 .
$$

This completes the proof of Theorem 3.2(iii).

4. Modular EQUATIONS OF DEgREE $n$ AND RELATIONS BETWEEN $c(q)$ AND $c\left(q^{n}\right)$

To briefly define a modular equation, we first write as usual

$$
(a)_{k}=\frac{\Gamma(a+k)}{\Gamma(a)} \text {. }
$$

We say that the modulus $\beta$ has degree $n$ over the modulus $\alpha$ when

$$
n \frac{{ }_{2} F_{1}(1 / 2,1 / 2 ; 1 ; 1-\alpha)}{{ }_{2} F_{1}(1 / 2,1 / 2 ; 1 ; \alpha)}=\frac{{ }_{2} F_{1}(1 / 2,1 / 2 ; 1 ; 1-\beta)}{{ }_{2} F_{1}(1 / 2,1 / 2 ; 1 ; \beta)}
$$

where

$$
{ }_{2} F_{1}(a, b ; c ; x)=\sum_{k=0}^{\infty} \frac{(a)_{k}(b)_{k}}{(c)_{k} k !} \quad x^{k}, \quad|x|<1 .
$$

A modular equation of degree $\mathrm{n}$ is an equation relating $\alpha$ and $\beta$ which is induced by (4.1).

THEOREM 4.1 . If

$$
q=\exp \left(-\pi \frac{{ }_{2} F_{1}(1 / 2,1 / 2 ; 1 ; 1-\alpha)}{{ }_{2} F_{1}(1 / 2,1 / 2 ; 1 ; \alpha)}\right)
$$

thèn

$$
\alpha=1-(2 c(q)-1)^{4}
$$

Proof: We have

$$
c(q)=\frac{1}{2}\left(1+\frac{\phi(-q)}{\phi(q)}\right) .
$$


From [4, p. 100, Entry 5] we know that the identity (4.2) implies that

$$
\alpha=1-\frac{\phi^{4}(-q)}{\phi^{4}(q)} .
$$

Using (4.3) and (4.4), we find that

$$
(2 c(q)-1)^{4}=1-\alpha,
$$

that is,

$$
\alpha=1-(2 c(q)-1)^{4} .
$$

Now, let $\alpha$ and $q$ be related by (4.2). If $\beta$ has degree $n$ over $\alpha$, then Theorem 4.1 gives us

$$
\beta=1-\left(2 c\left(q^{n}\right)-1\right)^{4}
$$

Hence by (4.5), (4.6) and any given modular equation of degree $n$, we can obtain a relation between $c(q)$ and $c\left(q^{n}\right)$. We illustrate this with $\mathrm{n}=3$ and $\mathrm{n}=4$.

Corollary 4.2. Let $u=c(q), v=c\left(q^{3}\right)$ and $w=c\left(q^{4}\right)$. Then,

(i) $\left(u^{2}+v^{2}\right)^{2}-12 u v(u+v)^{2}-4 u v[(1+2 u v)(2 u v-3(u+v))+1]=0$, and

(ii) $u=\frac{1}{1+\sqrt[4]{1-(2 w-1)^{4}}}$.

Proof of (i): When $\beta$ has degree 3 over $\alpha$, we have $[4$, Entry 5(ii), p. 230]

$$
(\alpha \beta)^{1 / 4}=1-((1-\alpha)(1-\beta))^{1 / 4}
$$

From (4.5) and (4.6), we have

$$
(2 c(q)-1)^{4}=1-\alpha
$$

and

$$
\left(2 c\left(q^{3}\right)-1\right)^{4}=1-\beta .
$$

Substituting these in (4.7), we obtain,

$$
\left(1-(2 u-1)^{4}\right)\left(1-(2 v-1)^{4}\right)=[1-(2 u-1)(2 v-1)]^{4} .
$$

On simplification, we deduce that

$$
\left(u^{2}+v^{2}\right)^{2}-12 u v(u+v)^{2}-4 u v[(1+2 u v)(2 u v-3(u+v))+1]=0 .
$$


Proof of (ii): When $\beta$ has degree 4 over $\alpha$, we have from [4, Equation (24.22) p. 215]

$$
\beta=\left(\frac{1-(1-\alpha)^{1 / 4}}{1+(1-\alpha)^{1 / 4}}\right)^{4}
$$

Also we have,

$$
1-\alpha=(2 u-1)^{4}
$$

and

$$
\beta=1-(2 w-1)^{4}
$$

Using (4.9) in (4.8) we obtain

$$
\beta^{1 / 4}=\frac{1-(2 u-1)}{1+(2 u-1)}
$$

which implies

$$
\beta^{1 / 4}=\frac{1-u}{u}
$$

Using (4.10) in (4.11) we get the required result.

\section{EXPLICIT FORMULAS FOR THE EVALUATIONS OF $c(q)$}

Let $q_{n}=e^{-\pi \sqrt{n}}$ and let $\alpha_{n}$ denote the corresponding value of $\alpha$ in (4.2). Then applying Theorem 4.1 , we get,

$$
c\left(e^{-\pi \sqrt{n}}\right)=\frac{\sqrt[4]{\left(1-\alpha_{n}\right)}+1}{2}
$$

It is known that from $\left[4\right.$, p. 97] that $\alpha_{1}=1 / 2, \alpha_{2}=(\sqrt{2}-1)^{2}$ and $\alpha_{4}=(\sqrt{2}-1)^{4}$. Hence by using (5.1) we deduce that

$$
\begin{aligned}
c\left(e^{-\pi}\right) & =\frac{\sqrt[4]{1 / 2}+1}{2}, \\
c\left(e^{-\pi \sqrt{2}}\right) & =\frac{\sqrt[4]{2(\sqrt{2}-1)}+1}{2},
\end{aligned}
$$

and

$$
c\left(e^{-2 \pi}\right)=\frac{\sqrt[4]{12 \sqrt{2}-16}+1}{2}
$$


Theorem 5.1. Let the Ramanujan-Weber Class invariants be defined by

$$
G_{n}:=2^{-1 / 4} q_{n}{ }^{-1 / 24}\left(-q_{n} ; q_{n}{ }^{2}\right)_{\infty}
$$

and

$$
g_{n}:=2^{-1 / 4} q_{n}{ }^{-1 / 24}\left(q_{n} ; q_{n}^{2}\right)_{\infty},
$$

where $q_{n}=e^{-\pi \sqrt{n}}$ and let $p=G_{n}{ }^{12}$ and $p_{1}=g_{n}{ }^{12}$. Then,
(i) $c\left(e^{-\pi \sqrt{n}}\right)=\frac{\sqrt[4]{\left(p+\sqrt{p^{2}-1}\right) /(2 p)}+1}{2}$, and
(ii) $c\left(e^{-\pi \sqrt{n}}\right)=\frac{\sqrt[4]{2 p_{1}\left(\sqrt{p_{1}^{2}+1}-p_{1}\right)}+1}{2}$.

ProOF OF (i): Since from [7],

$$
G_{n}=\left(4 \alpha_{n}\left(1-\alpha_{n}\right)\right)^{-1 / 24}
$$

we deduce that

$$
\alpha_{n}=\frac{1}{(\sqrt{p(p+1)}+\sqrt{p(p-1)})^{2}} .
$$

Using (5.5) in (5.1)we get the required result.

Proof of (ii): Also from [7], we have,

$$
\frac{1}{\left.\sqrt{(} \alpha_{n}\right)}-\sqrt{\left(\alpha_{n}\right)}=2 g_{n}^{12}
$$

Hence,

$$
\sqrt{\left(\alpha_{n}\right)}=\sqrt{\left(p_{1}^{2}+1\right)}-p_{1}
$$

Using (5.6) in (5.1), we complete the proof.

EXAmples. Let $n=1$. Since $G_{1}=1$, Theorem 5.1 yields

$$
c\left(e^{-\pi}\right)=\frac{\sqrt[4]{1 / 2}+1}{2} .
$$

Let $n=2$. Since $g_{2}=1$, Theorem 5.1 yields

$$
c\left(e^{-\pi \sqrt{2}}\right)=\frac{\sqrt[4]{2(\sqrt{2}-1)}+1}{2},
$$


Using $G_{3}^{12}=2$ in Theorem 5.1, we obtain

$$
c\left(e^{-\pi \sqrt{3}}\right)=\frac{\sqrt[4]{(2+\sqrt{3}) / 4}+1}{2} .
$$

\section{REFERENCES}

[1] C. Adiga, B.C. Berndt, S. Bhargava and G.N. Watson, Ramanujan's second notebook: Theta-functions and q-series (Chapter 16) Memoir no. 315 (Amer. Math. Soc., Providence, R.I., 1985).

[2] C. Adiga, K.R. Vasuki and M.S. Mahadeva Naika, 'Some new explicit evaluations of Ramanujan's cubic continued fraction', New Zealand J. Math. 31 (2002), 1-6.

[3] C. Adiga, K.R. Vasuki and K. Shivashankara, 'Some Theta-function identities and new explicit evaluations of Rogers-Ramanujan continued fraction', Tamsui Oxf. J. Math. Sci. 18 (2002), 101-117.

[4] B.C. Berndt, Ramanujan's Notebooks, Part III (Springer-Verlag, New York, 1991).

[5] B.C. Berndt and H.H. Chan, 'Some values of the Rogers-Ramanujan continued fraction', Canad. J. Math. 47 (1995), 897-914.

[6] H.H. Chan, 'On Ramanujan's cubic continued fraction', Acta Arith. 73 (1995), 343-355.

[7] H.H. Chan and S.S. Huang, 'On the Ramanujan-Göllnitz-Gordon continued fraction', Ramanujan J. 1 (1997), 75-90.

[8] K.G. Ramanathan, 'On the Rogers-Ramanujan continued fraction', Proc. Indian Acad. Sci. Math. Sci 93 (1984), 67-77.

[9] K.G. Ramanathan, 'Ramanujan's continued fraction', Indian J. Pure Appl. Math. 16 (1985), 695-724.

[10] K.G. Ramanathan, 'Some applications of Kronecker's limit formula', J. Indian. Math. Soc. (N.S.) 52 (1987), 71-89.

[11] S. Ramanujan, Notebooks (2 volumes) (Tata Institute of Fundamental Research, Bombay, 1957).

[12] S. Ramanujan, Collected papers (Chelsea, New York, 1962).

[13] S. Ramanujan, The lost notebook and other unpublished papers (Narosa Publishing House, New Delhi, 1988).

Department of Studies in Mathematics

University of Mysore

Manasagangotri

Mysore-570006

India

e-mail: c_adiga@hotmail.com
Department of Studies in Mathematics University of Mysore

Manasagangotri

Mysore-570006

India

e-mail: ani_nie@indiatimes.com 